\title{
Androgens, QT, sex and ventricular repolarization: a double- edged sword
}

\author{
Melissa Y.Y. Moey, M.D., MSc ${ }^{1}$, Marie Wilkin, M.D., Ph.D' ${ }^{2}$, Estelle Gandjbackhch, M.D., Ph.D. ${ }^{2}$, Anne Bachelot, \\ M.D., Ph.D. ${ }^{3}$, Baptiste Abbar, M.D. ${ }^{4}$, Bruno Pinna, M.D. ${ }^{4}$, Jean-Marc Simon, M.D. ${ }^{5}$, Christian Funck-Brentano, \\ M.D., Ph.D. ${ }^{4}$, and Joe-Elie Salem, M.D., Ph.D. ${ }^{4,6 *}$
}

1 Department of Cardiovascular Disease, Vidant Medical Center/East Carolina University, Greenville, North Carolina, USA; moeym16@ecu.edu

2 Department of Cardiology, Arrhythmia unit, Sorbonne Université, INSERM, AP-HP, Pitié-Salpêtrière Hospital, F-75013 Paris, France.; marie.wilkin24@gmail.com, estelle.gandjbakhch@aphp.fr

3 AP-HP, Pitié-Salpêtrière Hospital, IE3M, Department of Endocrinology and Reproductive Medicine, and Centre de Référence des Maladies Endocriniennes Rares de la croissance et Centre des Pathologies gynécologiques Rares; Sorbonne Universités, F-75013 Paris, France; anne.bachelot@aphp.fr

4 Sorbonne Université, GRC n5, Oncotype-Uro, Department of Oncology Radiotherapy, Assistance PubliqueHôpitaux de Paris (APHP), Pitié-Salpêtrière Hospital, F-75013 Paris, France; baptiste.abbar@aphp.fr, Bruno.pinna@aphp.fr $\underline{\text { christian.funck-brentano@aphp.fr }}$

5 Sorbonne Université, INSERM CIC Paris-Est (CIC-1901), AP-HP.Sorbonne Université, Pitié-Salpêtrière Hospital, Department of Pharmacology, UNICO-GRECO Cardio-Oncology Program, F-75013 Paris, France; jean-marc.simon@aphp.fr

6 Departments of Medicine and Pharmacology, Cardio-Oncology program, Vanderbilt University Medical Center, Nashville, Tennessee, USA; joe-elie.salem@aphp.fr

* Correspondence: joe-elie.salem@aphp.fr

\begin{abstract}
The prevalence and incidence of cardiac pro-arrhythmic disorders are often influenced by sex due to specific effects on the QT interval. Androgens shorten QT, which may be protective against acquired long QT syndromes and their related arrhythmias in men such as torsade de pointes (TdP). On the other hand, androgens can potentiate Brugada and early repolarization syndromes, which are most prevalent in men. In this case series, we highlight four male patients with aborted SCD in the setting of abnormal testosterone status; two patients with TdP in a setting of testosterone deprivation (of which one drug-induced) and 2 patients with ventricular fibrillation associated with exogenous androgenic booster (Tribulus terrestris) intake. From this case series, we review the current available literature of the effects of androgen as a double-edged sword on the QTc interval and emphasize the importance of QTc monitoring in this subset of patients.
\end{abstract}

Keywords: testosterone, androgens, QT, Torsade de pointes, ventricular repolarization

\section{Introduction}

The prevalence and incidence of cardiac pro-arrhythmic disorders are often influenced by sex due to specific effects on the QT interval. The normal QT interval corrected for heart rate (QTc) ranges between 350 to 450 milliseconds (ms) in men and 360 to $460 \mathrm{~ms}$ in women. Values below and above these intervals are suggestive of underlying channelopathies [1].

Acquired long QT syndrome (LQTS), which is mainly drug-induced (DiLQTS), is often secondary to defects in ventricular repolarizing currents (delayed rectifier potassium current, rapid $[\mathrm{IKr}]$ ) associated with excessive action potential duration (APD) lengthening and is most prevalent in women [1,2]. In contrast, Brugada syndrome (BrS) and early repolarization syndrome (ERS) are more frequent in men and are due to activation of polarizing currents associated with excessive APD shortening and eventually shorter QT duration (for ERS) as compared to a control population [3,4]. LQTS, BrS and ERS favor different forms of malignant ventricular arrhythmias - for example, torsade de 
pointes (TdP) for LQTS, and ventricular tachycardia (VT) for BrS and ERS that may lead to ventricular fibrillation (VF), and if sustained, sudden cardiac death (SCD) [1-4]. This sex specific prevalence of LQTS, BrS and ERS is partly explained by the fact that androgens shorten APD and QT duration, while its deprivation has opposite effects $[5,6]$.

In this case series, we highlight four male patients with aborted SCD in the setting of abnormal testosterone status; two patients with TdP in a setting of testosterone deprivation (of which one drug-induced) and 2 patients with VF associated with exogenous androgenic booster (Tribulus terrestris) intake [7]. From these case series, we review the current available literature of the complex relationship between androgens and QTc interval, emphasizing the importance of QTc monitoring and risk of ventricular arrhythmias in this subset of patients.

\section{Case Series Presentation}

Case 1: A 91 y.o. man with known normal QTc Friderica (QTcF) [1] (QTcF=420ms) and past medical history of dyslipidemia, asymptomatic paroxysmal grade 2 atrioventricular block (AVB), hypertension, subdural hematoma, arthrosis and prostatic adenoma developed a syncopal TdP in the setting of acute bradycardia complicating a paroxysmal complete AVB associated with an extreme QTc prolongation (QTcF>600 ms; Figure 1). At the time of the event, he was not taking any QTc prolonging drug with known risk of TdP according to the Crediblemeds website (https://crediblemeds.org/). A transthoracic echocardiogram (TTE) showed a preserved left ventricular ejection fraction (LVEF) and troponin and potassium levels were normal. Hormonal assays showed a profound hypogonadism due to a primary testicular failure with very low total testosterone level $(0.6 \mathrm{ng} / \mathrm{ml}$; reference values: [1.9-7.4 ng/ml]) and increased follicle stimulating hormone (FSH) and luteinizing hormone (LH) levels (48.4 IU/1, [1.5-12.4 IU/1]; 43.7 IU/1,[1.7-8.6 IU/1]; respectively). The patient received isoproterenol and intravenous magnesium, followed urgently by implantation of a permanent pacemaker with a ventricular pacing rate set at $80 \mathrm{bpm}$. Subsequently, testosterone supplementation was started and the patient remained dependent on ventricular pacing, precluding a reliable QTc measurement. At 9 months follow-up, there was no recurrence of TdP. Genetic screening performed for rare variants in KCNQ1, KCNH2, SCN5A, plus 12 other minor genes involved in congenital LQTS was negative.

Case 2: A 72 y.o. man with past medical history of hypertension, dyslipidemia, moderate mitral regurgitation secondary to mitral valve prolapse was known for a prostate cancer treated by prostatectomy in 2014 followed by radiotherapy. In 2018, luteinizing hormone (LH) therapy (goserelin 10.8mg every 12 weeks) was started for a biological recurrence with increased PSA $(7.9 \mathrm{ng} / \mathrm{mL}$; baseline of $0.2 \mathrm{ng} / \mathrm{mL})$. On this treatment, ECG was normal with a QTcF of $413 \mathrm{~ms}$. Two years later, the prostate cancer evolved into a castration resistant metastatic state (PSA:13.3ng/mL) requiring the addition of enzalutamide (a nonsteroidal antiandrogen, $160 \mathrm{mg} /$ day, oral) to goserelin. One month after initiation of enzalutamide (Figure 2A), during a routine follow-up, the ECG showed a prolonged QTcF 600ms and repeat echocardiogram demonstrating severe mitral regurgitation with preserved left ventricular ejection fraction (LVEF). A coronary computed tomography (CT) scan was normal. An ECG Holter recording identified 9000 polymorphic premature ventricular contractions/day (with doublets, triplets, quadruplets and R-on-T phenomenon) with prolonged QTc. He was subsequently hospitalized, where he had several symptomatic spontaneously reversible TdP episodes (Figure 2B). QTc remained above $550 \mathrm{~ms}$ despite normalization of potassium and magnesium blood levels. It was decided to implant a pacemaker to allow for set extrinsic pacing (rate of 80$90 \mathrm{bpm}$ ) and avoidance of TdP recurrence while continuing enzalutamide as it was controlling prostate cancer. A few weeks later, the patient presented with several episodes of syncope. While admitted to the hospital, he had a VF cardiac arrest with subsequent achievement of return of spontaneous circulation after external electrical cardioversion. 
Post-arrest ECG showed normal sinus rhythm, rate of $90 \mathrm{bpm}$ and a prolonged QTcF of $672 \mathrm{~ms}$ (Figure 2C). Echocardiography showed a preserved left ventricular ejection fraction (LVEF) with previously observed severe mitral regurgitation. Troponin and potassium levels were normal. Enzalutamide was stopped, and QTcF progressively shortened to near-normal duration two weeks after follow-up (QTcF:480-500msec, Figure 2D). Mitral valve repair was subsequently pursued one month later with implantation of a defibrillator, and introduction of beta-blockers. At 5 months of follow-up, there was no recurrence of TdP. The patient was continued on goserelin and QTc remained in the slightly prolonged range $(480-500 \mathrm{~ms})$ but prostate cancer was still controlled (PSA: 0.37 $\mathrm{ng} / \mathrm{mL})$.

Case 3: Four months after initiating Tribulus terrestris [7] (Tribooster ${ }^{\circledR}$, BioTech, USA; 1500mg/pill, one pill every other day), a 32 y.o. man with no personal medical or family history of cardiac disease presented with an aborted VF cardiac arrest (Figure 3) following a total of 7 electrical cardioversions over a few hours. The patient was not taking any other concurrent medications or exogenous supplements. After restoration of sinus rhythm, amiodarone was started as well as therapeutic hypothermia and pressor support. Initial evaluation showed a depressed LVEF of $30 \%$, high potassium levels (6 $\mathrm{mmol} / \mathrm{l}$ ) and increased troponin level (peak troponin: $2541 \mathrm{ng} / \mathrm{L}$; upper normal limit: 50 ng/L) with an ECG compatible with BrS (Figure 2B). Coronary angiogram subsequently performed was normal. Amiodarone was replaced by isoproterenol infusion and oral quinidine (300mg BID) in this context of electrical storm with possible underlying BrS. VF did not recur with this anti-arrhythmic strategy. Cardiac magnetic resonance imaging showed a normalization of LVEF with an intra-septal late gadolinium enhancement 9 days after the index event. Troponin levels normalized within 2 weeks. Ten days after the index event, after withholding quinidine, ajmaline test was negative and ECG showed features of ERS with QTcF measured between 360-400 ms (Figure 2C). Concomitantly, hormonal assays showed a slightly supra-physiological total testosterone level $(8.5 \mathrm{ng} / \mathrm{ml}$, reference range: [1.9-7.4 $\mathrm{ng} / \mathrm{ml}])$ with normal TSH $(1.23 \mathrm{mUI} / \mathrm{l}$, reference range: $0.27-4.0 \mathrm{mUI} / \mathrm{l}$ ), normal FSH and LH levels (1.6 IU/1 and $7.5 \mathrm{IU} / 1$; respectively). The patient was subsequently discharged on quinidine $300 \mathrm{mg}$ per os BID with no recurrence of VF at 3 months of follow up. He underwent implantation of a subcutaneous implantable cardioverter defibrillator (ICD). Genetic screening performed for rare variants in SCN5A, KCNJ8, CACNA1C, CACNB2, CACNA2D1 involved in congenital $\mathrm{BrS} / \mathrm{ERS}$ was negative.

Case 4: Through a search of the French national nutrivigilance database for other reports of SCD or ventricular arrhythmias suspected to be associated with Tribulus terrestris intake, we discovered a 21 y.o. man with aborted SCD 2 weeks following the start of a compound containing Tribulus terrestris (PLZ+ $₫$, Abo Factory). He was an active smoker without any past medical history or chronic medication use. A computed tomography (CT) angiogram of the chest ruled out a pulmonary embolism, and coronary angiogram and cardiac magnetic resonance imaging did not identify any coronary or myocardial abnormalities, respectively. Initial TTE showed an LVEF of $40 \%$, with excessive tissues on the tricuspid and mitral valves, suggestive of Barlow disease. ECG on admission showed paroxysmal atrial fibrillation and pharmacological tests subsequently performed were unrevealing (isoprenaline, ajmaline, adrenaline, adenosine triphosphate) for an underlying channelopathy. Patient improved favorably after withdrawal of PLZ $+\circledR$ and was subsequently discharged after implantation of an ICD. Follow-up ECG traces showed that he was in sinus rhythm with QTc values between 380 and $400 \mathrm{~ms}$. 


\subsection{Figures}

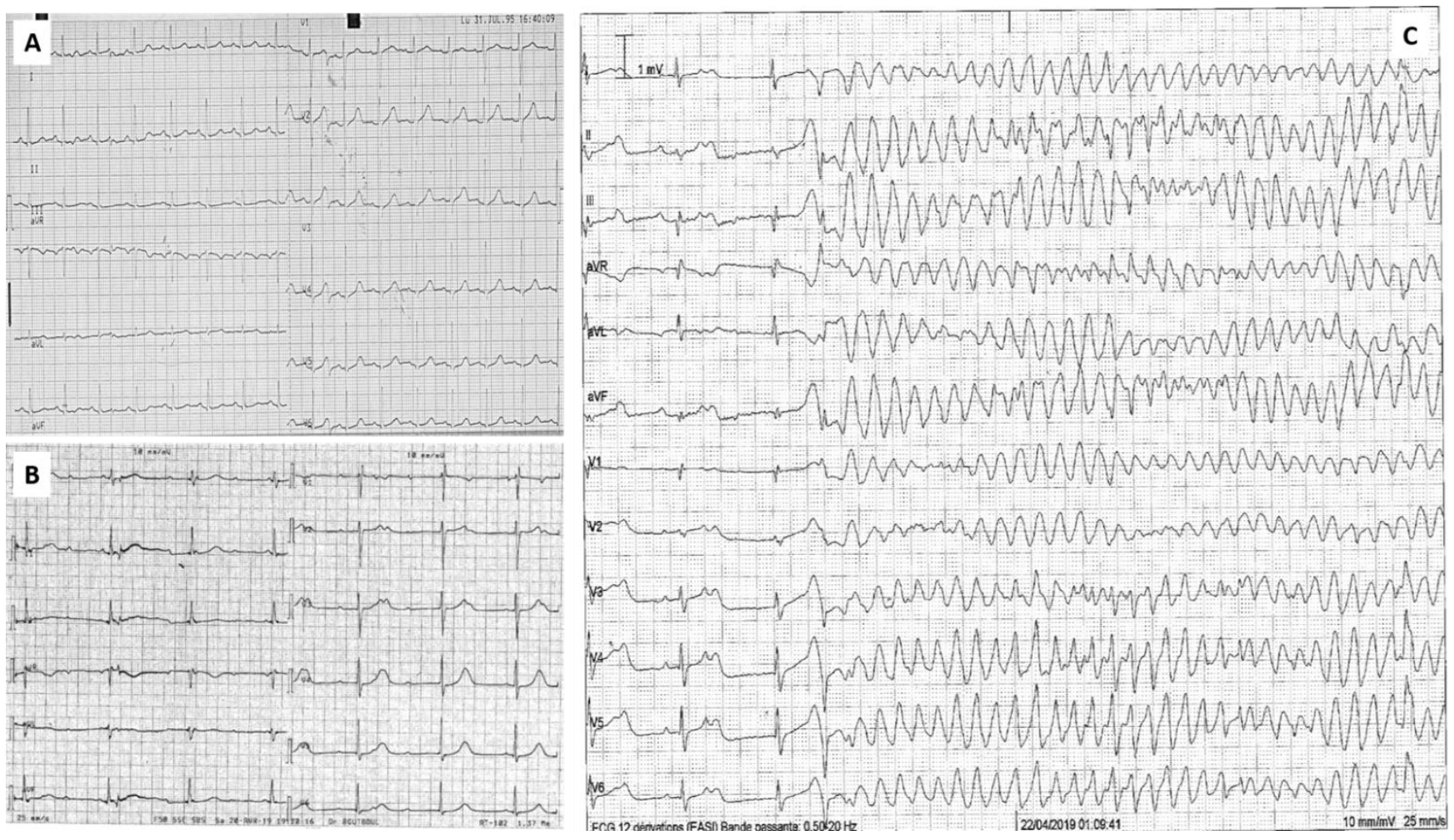

Figure 1. Electrocardiograms (ECG) of a 91 y.o. man who presented with a syncopal torsade de pointes (TdP) episode complicated by a complete atrioventricular block in the setting of hypogonadism. ECG showing a normal QTcF value (420 ms) approximatively 20 years before TdP (A). ECG in complete atrioventricular block, showing an extremely prolonged QTcF $>600 \mathrm{~ms}$ with T-wave notching (B) preceding the TdP (C).

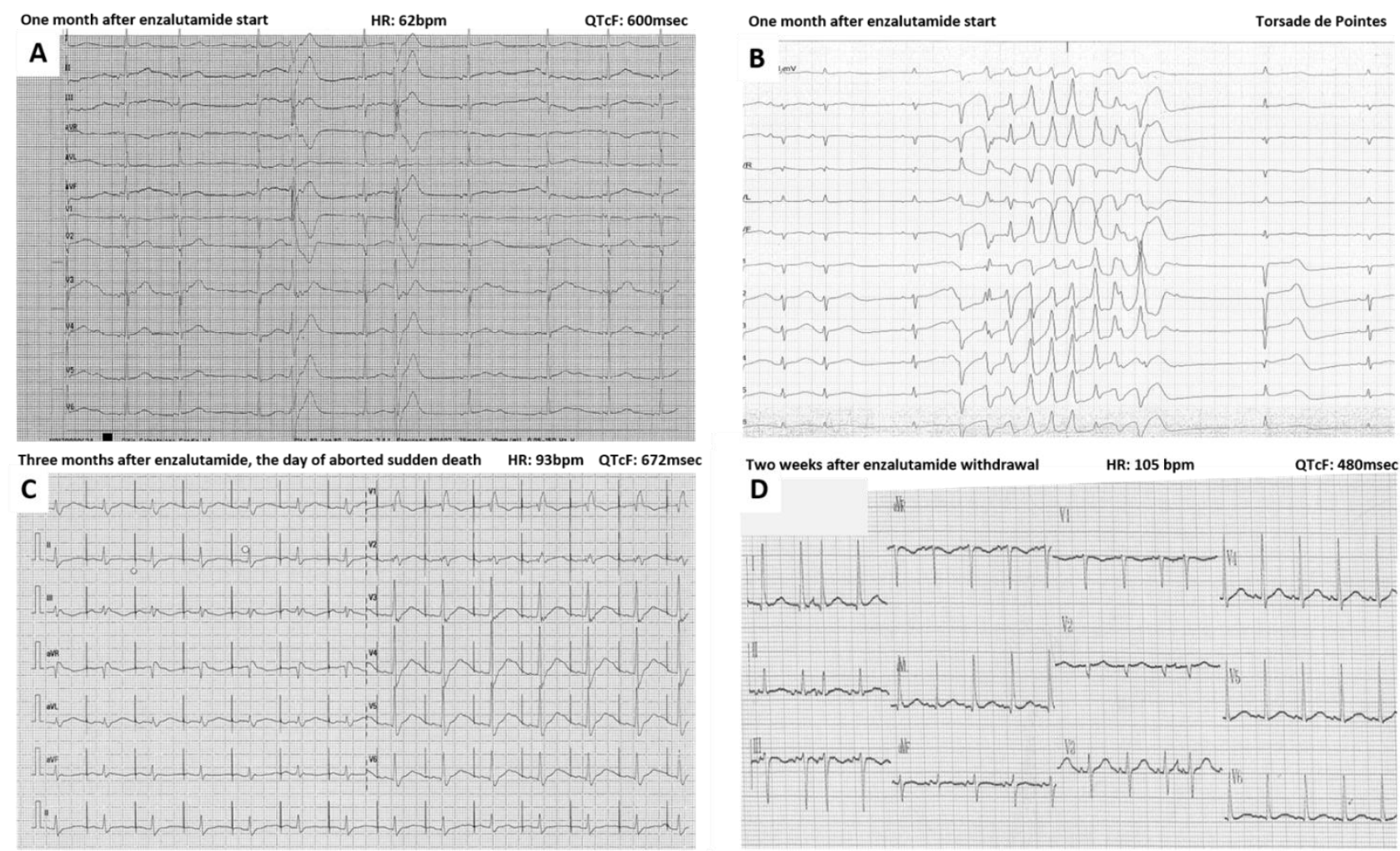

Figure 2. Electrocardiograms (ECG) of a 72 y.o. man with prostate cancer who presented with a QTcF prolongation around 600 $\mathrm{ms}(\mathrm{A})$ and asymptomatic episodes of spontaneously reversible torsade de pointes (B) one month after introduction of 
enzalutamide, an androgen receptor antagonist. Three months after enzalutamide start, despite a rapid ventricular pacing between 80-90bpm (C), the patient presented an aborted sudden death episode. Enzalutamide was withdrawn and QTcF decreased around 480-500msec (D) 2 weeks after liable drug withdrawal.
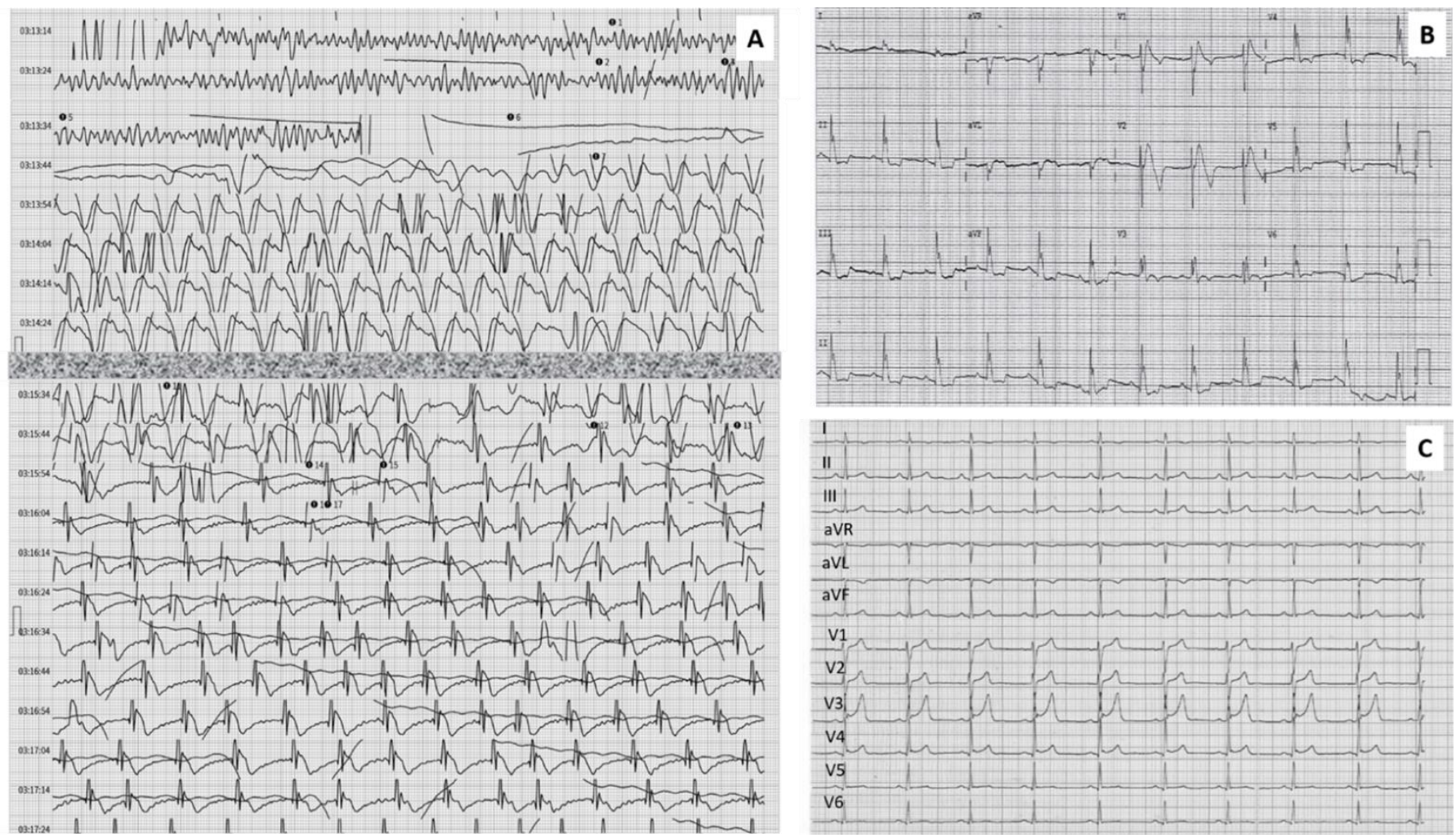

Figure 3. Electrocardiograms (ECG) of a 32 y.o. man who presented as an aborted sudden cardiac death associated with Tribulus terrestris intake (androgenic booster). Defibrillator strip at the time of the first ventricular fibrillation treated by electrical cardioversion (A). ECG in sinus rhythm 3 hours after the first ventricular event while in hypothermia showing J-Osborn waves and repolarization abnormalities compatible with BrS (B). ECG in sinus rhythm 8 days after the first ventricular event, showing early repolarization features $(\mathrm{C})$.

\section{Discussion}

This case series highlights the likely complex relationship (U-shape) between androgen levels in men and the risk for ventricular arrhythmias and SCD. In Case 1 and 2, both patients developed TdP in the setting of acquired LQTS associated with hypogonadism due to endogenous age-related testicular insufficiency and exogenous hypogonadism induced by antiandrogenic therapy for prostate cancer, respectively. In contrast with the previous cases, patients in Case 3 and 4 were using the exogenous androgen booster (Tribulus terrestris) and experienced VF, Case 3 being diagnosed with ERS/BrS. These data support the concept that androgen levels should be monitored in men presenting with ventricular arrhythmias and eventually corrected accordingly, depending on the electrocardiographic presentation.

Large studies have shown that DiLQTS and TdP are more prevalent in women $(\approx 2 / 3)$ than in men [8]. Although there are multiple possible mechanisms for shorter QTc and lower TdP risk in men versus women [8], this can be in part explained by circulating testosterone levels since hypogonadism or androgen deprivation therapies (ADT) blunt the 10-20msec difference in QTc between men and women [2]. In a case-series of seven consecutive men prospectively admitted for TdP, we showed that all had hypogonadism and that normalization of testosterone levels shortened QTc and prevented recurrence of TdP [6]. The effects of testosterone on the QT interval were also demonstrated in a small randomized controlled study of men where ibutilide-induced QTcF prolongation was reversed with subdermal testosterone in comparison to placebo [8]. Similarly, in extreme 
cases with severely decreased total, free and bioavailable testosterone levels which was demonstrated in a retrospective analysis from a TdP registry, patients receiving ADT, particularly leuprolide and bicalutamide, for prostate cancer had prolonged QTc (QTc 530$620 \mathrm{~ms}$ ) and suffered TdP [9]. In a recent study among 149 patients who were receiving GnRH analogues as part of treatment for prostate cancer, QTc interval was significantly prolonged during therapy in comparison to baseline (439 $\pm 31 \mathrm{~ms}$ vs $416 \pm 27 \mathrm{~ms}$, respectively) [10]. The authors observed among 2 patients who experienced TdP and VF, QTc was prolonged $>80 \mathrm{~ms}$ suggesting that a QTc interval $>50 \mathrm{~ms}$ may be considered a predictor of $\mathrm{TdP} / \mathrm{VF}$. Thus further emphasizing the importance of QTc monitoring in patients receiving $\mathrm{GnRH}$ analogues for prostate cancer [10].

For women, this finding is also relevant in the form of different androgenic properties of oral contraceptive pills, drospirenone which has antiandrogenic effects versus levonorgestrel which has androgenic properties, also has significant effects on drug-induced prolongation of ventricular repolarization. In women with sotalol-induced prolonged QTc, there was significant lengthening of QTc in these patients with concomitant drospirenone use (baseline QTc of $394 \pm 15.2 \mathrm{~ms}$ with an increase by $31 \pm 12.6 \mathrm{~ms}$ ) than in patients taking levonorgestrel (baseline QTc of $392 \pm 14.2 \mathrm{~ms}$ with an increase of $24.2 \pm 13.7$ $\mathrm{ms}$ ) or sotalol only (baseline QTc of $393 \pm 16.9 \mathrm{~ms}$ with an increase in $24.6 \pm 12.5 \mathrm{~ms}$ ). This was further corroborated by analysis of a large pharmacovigilance database which observed more reports of diLQTS and ventricular arrhythmias with drospirenone than with levonorgestrel [5].

In comparison, male sex, higher testosterone levels and use of androgen agonists are risk factors for ERS and BrS favoring VT and VF [11]. ERS and BrS are predominantly seen in younger men when compared to LQTS which has a strong female prevalence, that has been demonstrated to be related specifically to higher testosterone levels [12]. Brugada and Brugada were the first to describe the QTc interval and clinical phenotype in BrS and is generally perceived to be within normal gender specific range in patients with the SCN5A mutation, the gene that encodes the $\alpha$-subunit of the cardiac sodium channel leading to INa current [4]. A "coved" ST segment-J-point elevation with concave ST segment elevation merging into a negative or isoelectric symmetric T-wave typically observed in leads V1 and V2 of the 3rd or 2nd intercostal space, is the ECG pattern characteristic of type $1 \mathrm{BrS}$. A shorter QTc however has been observed in BrS with loss-of-function mutations in the genes encoding the cardiac L-type calcium channel [13]. Among 2,681 Japanese male atomic bomb survivors, patients with Brugada-like ECG patterns conferred a higher risk (relative risk [RR] of $6.47,95 \%$ CI 1.97-21.21, $\mathrm{p}=0.002$ ) of prostate cancer independent of radiation exposure, age and tobacco use, further suggesting that high levels of testosterone may contribute to BrS phenotype [14]. The direct effect of testosterone on BrS has also been shown by surgical castration and ADT in patients with prostate cancer which resulted in the disappearance of the classical BrS ECG phenotype, suggesting that testosterone may modulate the early phase of ventricular repolarization [15]. In ERS which is highly prevalent in the male population (90\%), the QTc is significantly shorter in males than in females and is suspected to be due to the androgenic effects of testosterone on the repolarization duration [3].

Originating from the plant family of Zygophyllaceae which has been used since ancient times for multitude effects particularly as an aphrodisiac, improvement in sexual dysfunction, anti-inflammatory and anti-oxidant properties, Tribulus terrestris is an overthe-counter nutritional herbal supplement advertised to increase male performance [7]. Several studies have demonstrated in different animal models that Tribulus terrestris increases the levels of testosterone as well as its active metabolite (dihydrotestosterone, DHT) and dehydroepiandrosterone sulphate [7]. In comparison to control primate subjects, primates who were administered Tribulus terrestis experienced a mean $51 \%$ and $32 \%$ increase in testosterone and DHT. In castrated rats receiving Tribulus terrestris, testosterone levels were increased by $25 \%$. The effect of testosterone and DHT increase is suspected to be secondary to the predominant steroidal glycoside (saponins) furostonal of Tribulus terrestris, protodioscin (PTN), which enhances androgen production through 
either direct hormonal receptor binding or alteration of the steroidal enzyme metabolism [7]. In patients with hypogonadism, PTN treatment has been shown to increase levels of testosterone and luteinizing hormones. Though the effects of Tribulus terrestris on ECG parameters has not been directly studied, we suspect that our patient in Cases 3 and 4 who suffered VF arrest was likely due to increased testosterone and DHT induced by Tribulus terrestris that effectively shortened QTc interval and increased the risk for VF of an underlying ERS/BrS.

Reviewed extensively elsewhere [2,11], exogenous testosterone and DHT can shorten QTc duration in vivo and decrease the APD in preclinical models. The ECG pattern of a longer repolarization duration (longer QT interval and lower T-wave amplitude) in women has been well established to differ from men as a result of the androgenic effect of testosterone. In men with hypogonadism who received exogenous intramuscular injection of testosterone, there was a significant negative linear relationship observed with QT interval and testosterone level whereby QTc was longer (median QTc $363 \mathrm{~ms}$, IQR 357 to $384 \mathrm{~ms}$ ) during lower levels of testosterone and shorter (median QTc $352 \mathrm{~ms}$, IQR 340 to $363 \mathrm{~ms}$ ) during higher levels of testosterone [16]. In a larger study that looked at the ECG parameters of two randomized controlled clinical trials assessing testosterone replacement in opioid-induced androgen deprivation patients (Testosterone and Pain, TAP) and the effect on progression of subclinical atherosclerosis in older men (Testosterone Effects on Atherosclerosis in Aging Men, TEAAM), testosterone reduced the age-related increase in QTc duration in men [17]. Specifically, within the TAP trial, change in QTc was negatively associated with change in total testosterone $(r=-0.24, p=0.036)$ and in the TEAAM trial, there was a decrease in QTc by $-6.3 \mathrm{~ms}(\mathrm{p}<0.001)[16]$.

The effects of exogenous chronic anabolic-androgenic steroid (AAS) use on the other hand, such as oxymetholone, stanozolol, and nandrolone, is more complex as they have variable effects on the ECG pattern due to their different androgenic and anabolic properties (anabolic-to-androgen ratio) and interactions with gonadotropins, which is dependent on the chemical structure of the compound studied [11]. AAS are testosterone derivatives with the primary function of a higher anabolic-to-androgen ratio; for example, oxymetholone has an anabolic-to-androgen ratio of 9 while stanozolol has an anabolic-toandrogen ratio of 30, in comparison to testosterone of 1 [18]. Thus, chronic AAS use can cause significant increase in non-testosterone androgen plasma levels which results in a negative feedback mechanism on endogenous testosterone secretion by the testis due to suppression of the hypothalamic-pituitary-gonadal axis. QTc was even found prolonged in some studies of chronic AAS in male bodybuilders [11].

The data on the mechanism of the effect of hormonal changes specific to gender on the ventricular repolarization and APD have not entirely been elucidated, however have been summarized in recent reviews [2,11]. Specifically, the change in morphology and shortening of the ventricular APD was shown to be related to higher ITo (transient outward potassium current) density in the male ventricular epicardial cells than in female cardiomyocytes in dogs [19]. In addition, in rodent-based preclinical studies, testosterone has been suggested to shorten the APD by enhancement of the repolarizing IKr and IKs (delayed rectifier potassium current, slow) currents and by decreasing the depolarizing L-type calcium current [20].

Exogenous hormonal suppression therapy has been a mainstay for cancer treatment in prostate cancer. Enzalutamide which is a competitive inhibitor of androgen binding at the androgen receptor has been shown to be associated with DiLQTs and high fatality rates. Gheorghe and colleagues observed a significant QTc prolongation of $32.6 \pm 43.6 \mathrm{~ms}$ in patients receiving combination enzalutamide and degarelix in comparison to patients on degarelix monotherapy. Although this was a small study of 35 patients, there were 6 patients among the group receiving enzalutamide and degarelix with more than 60ms QTc prolongation felt to be secondary to concomitant history of prior myocardial infarction [21]. In a prior translational study by our group, enzalutamide was found to significantly prolong the APD in cardiomyocytes from induced pluripotent stem cells from men [22]. With exposure to both acute (15 minutes) and chronic (5 hours) enzalutamide, the APD 
was significantly prolonged and corresponded to an inhibition in IKr and enhancement of the late sodium current ( $\mathrm{I}_{\mathrm{Na}-\mathrm{L}}$ ), which was abrogated by DHT administration [22].

\section{Conclusions}

In summary, this case series is concordant with our previous findings which support the concept of the role of androgen levels in the setting of channelopathies and the risk for malignant arrhythmias in men. This emphasizes the need for further evaluation of androgen levels in men of all ages who present with aborted SCD to promptly identify and reverse hypo- or hyperfunctioning exogenous or endogenous androgen states. Interplay of sex hormones levels with the clinical penetrance of intra- and inter-variability of genetic channelopathies affecting the main cardiac ionic currents deserve further scrutiny.

Author Contributions: Conceptualization: J-E.S. Methodology, Investigation: J-E.S. Original draft preparation, writing: M.M., M.W. Reviewing and editing: E.G., A.B., B.A, B.P., J-M.S., C.F.B., J-E.S. Supervision: J-E.S.

Funding: This research received no external funding.

Institutional Review Board Statement: Not applicable.

Informed Consent Statement: Not applicable.

Acknowledgments: None.

Conflicts of Interest: The authors declare no conflict of interest

\section{References}

1. Roden DM. Drug-Induced Prolongation of the QT Interval. New England Journal of Medicine. 2004;350(10):1013-1022. doi:10.1056/NEJMra032426

2. Barber M, Nguyen LS, Wassermann J, Spano JP, Funck-Brentano C, Salem JE. Cardiac arrhythmia considerations of hormone cancer therapies. Cardiovascular Research. 2019;115(5):878-894. doi:10.1093/cvr/cvz020

3. Panicker GK, Manohar D, Karnad DR, Salvi V, Kothari S, Lokhandwala Y. Early repolarization and short QT interval in healthy subjects. Heart Rhythm. 2012;9(8):1265-1271. doi:10.1016/j.hrthm.2012.03.046

4. Brugada P, Brugada J. Right bundle branch block, persistent ST segment elevation and sudden cardiac death: A distinct clinical and electrocardiographic syndrome. A multicenter report. Journal of the American College of Cardiology. 1992;20(6):1391-1396. doi:10.1016/0735-1097(92)90253-J

5. Salem JE, Dureau P, Bachelot A, et al. Association of oral contraceptives with drug-induced QT interval prolongation in healthy nonmenopausal women. JAMA Cardiology. 2018;3(9):877-882. doi:10.1001/jamacardio.2018.2251

6. Salem JE, Waintraub $X$, Courtillot $C$, et al. Hypogonadism as a reversible cause of torsades de pointes in men. Circulation. 2018;138(1):110-113. doi:10.1161/CIRCULATIONAHA.118.034282

7. Gauthaman K, Ganesan AP. The hormonal effects of Tribulus terrestris and its role in the management of male erectile dysfunction - an evaluation using primates, rabbit and rat. Phytomedicine. 2008;15(1-2):44-54. doi:10.1016/j.phymed.2007.11.011

8. Muensterman ET, Jaynes HA, Sowinski KM, et al. Effect of transdermal testosterone and oral progesterone on drug-induced QT interval lengthening in older men: a randomized, double-blind, placebo-controlled crossover-design study. Circulation 2019:140(13):1127-1129. doi: 10.1161/CIRCULATIONAHA.119.041395

9. Lazzerini PE, Bertolozzi I, Acampa M, et al. Androgen Deprivation Therapy for Prostatic Cancer in Patients With Torsades de Pointes. Frontiers in Pharmacology. 2020;11(May):1-11. doi:10.3389/fphar.2020.00684

10. Hasegawa K, Ito H, Kaseno K, et al. Impact of Medical Castration on Malignant Arrhythmias in Patients With Prostate Cancer. J Am Heart Assoc. 2021 Feb;10(5):e017267. doi: 10.1161/JAHA.120.017267

11. Salem JE, Alexandre J, Bachelot A, Funck-Brentano C. Influence of steroid hormones on ventricular repolarization. Pharmacology and Therapeutics. 2016;167:38-47. doi:10.1016/j.pharmthera.2016.07.005

12. Junttila MJ, Tikkanen JT, Porthan K, et al. Relationship between testosterone level and early repolarization on 12-lead electrocardiograms in men. Journal of the American College of Cardiology. 2013;62(17):1633-1634. doi:10.1016/j.jacc.2013.07.015

13. Antzelevitch C, Pollevick GD, Cordeiro JM, et al. Loss-of-function mutations in the cardiac calcium channel underlie a new clinical entity characterized by ST-segment elevation, short QT intervals, and sudden cardiac death. Circulation. 2007;115(4):442-449. doi:10.1161/CIRCULATIONAHA.106.668392

14. Haruta D, Matsuo K, Ichimaru S, et al. Men with Brugada-like electrocardiogram have higher risk of prostate cancer. Circ J. 2009 Jan;73(1):63-8. doi: 10.1253/circj.cj-08-0680

15. Ezaki K, Nakagawa M, Taniguchi Y, et al. Gender differences in the ST segment: Effect of androgen-deprivation therapy and possible role of testosterone. Circulation Journal. 2010;74(11):2448-2454. doi:10.1253/circj.CJ-10-0221 
16. Charbit B, Christin-Maître S, Démolis JL, Soustre E, Young J, Funck-Brentano C. Effects of Testosterone on Ventricular Repolarization in Hypogonadic Men. American Journal of Cardiology. 2009;103(6):887-890. doi:10.1016/j.amjcard.2008.11.041

17. Gagliano-Jucá T, Içli TB, Pencina KM, et al. Effects of Testosterone Replacement on Electrocardiographic Parameters in Men: Findings From Two Randomized Trials. J Clin Endocrinol Metab. 2017;102(5):1478-1485. doi: 10.1210/jc.2016-3669

18. Rahnema CD, Lipshultz LI, Crosnoe LE, Kovac JR, Kim ED. Anabolic steroid-induced hypogonadism: Diagnosis and treatment. Fertility and Sterility. 2014;101(5):1271-1279. doi:10.1016/j.fertnstert.2014.02.002

19. di Diego JM, Cordeiro JM, Goodrow RJ, et al. Ionic and cellular basis for the predominance of the Brugada syndrome phenotype in males. Circulation. 2002;106(15):2004-2011. doi:10.1161/01.CIR.0000032002.22105.7A

20. Abehsira G, Bachelot A, Badilini F, et al. Complex influence of gonadotropins and sex steroid hormones on QT interval duration. Journal of Clinical Endocrinology and Metabolism. 2016;101(7):2776-2784. doi:10.1210/jc.2016-1877

21. Gheorghe ACD, Ciobanu A, Hodorogea AS, et al, Gheorghe GS. Evolution of Electrocardiographic Repolarization Parameters During Antiandrogen Therapy in Patients with Prostate Cancer and Hypogonadism. Cardiovasc Toxicol. 2020 Aug;20(4):390-400. doi: 10.1007/s12012-020-09566-6

22. Salem JE, Yang T, Moslehi JJ, et al. Androgenic effects on ventricular repolarization a translational study from the international pharmacovigilance database to iPSC-cardiomyocytes. Circulation. 2019;140(13):1070-1080. doi:10.1161/CIRCULATIONAHA.119.040162 